\title{
Erratum to: Probing quantum gravity effects with quantum mechanical oscillators ${ }^{\star, \star \star}$
}

Eur. Phys. J. D (2020) 74: 178, https://doi.org/10.1140/epjd/e2020-10184-6

Michele Bonaldi ${ }^{1,2}$, Antonio Borrielli ${ }^{1,2}$, Avishek Chowdhury ${ }^{3,4}$, Gianni Di Giuseppe ${ }^{5,6}$, Wenlin $\mathrm{Li}^{5}$, Nicola Malossi ${ }^{5}$, Francesco Marino ${ }^{3,4, a}$, Bruno Morana ${ }^{7}$, Riccardo Natali ${ }^{5,6}$, Paolo Piergentili ${ }^{5,6}$, Giovanni A. Prodi ${ }^{2,8}$, Pasqualina M. Sarro ${ }^{7}$, Enrico Serra ${ }^{1,2,7}$, Paolo Vezio ${ }^{4,9}$, David Vitali ${ }^{3,5,6}$, and Francesco Marin ${ }^{3,4,9,10}$

1 Institute of Materials for Electronics and Magnetism, Nanoscience-Trento-FBK Division, 38123 Povo, Trento, Italy

2 Istituto Nazionale di Fisica Nucleare (INFN), Trento Institute for Fundamental Physics and Application, 38123 Povo, Trento, Italy

3 CNR-INO, L.go E. Fermi 6, 50125 Firenze, Italy

4 INFN, Sezione di Firenze, Via Sansone 1, 50019 Sesto Fiorentino, FI, Italy

5 School of Science and Technology, Physics Division, University of Camerino, Via Madonna delle Carceri, 9, 62032 Camerino, MC, Italy

6 INFN, Sezione di Perugia, Via A. Pascoli, 06123 Perugia, Italy

7 Laboratory of Electronic Components Technology and Materials (ECTM), Department of Microelectronics, Delft University of Technology, Feldmanweg 17, 2628 CT Delft, The Netherlands

8 Dipartimento di Fisica, Università di Trento, 38123 Povo, Trento, Italy

9 European Laboratory for Non-Linear Spectroscopy (LENS), Via N. Carrara 1, 50019 Sesto Fiorentino, FI, Italy

10 Dipartimento di Fisica e Astronomia, Università di Firenze, Via G. Sansone 1, 50019 Sesto Fiorentino, FI, Italy

Received 19 October 2020 / Accepted 19 October 2020

Published online 19 November 2020

(C) EDP Sciences / Società Italiana di Fisica / Springer-Verlag GmbH Germany, part of Springer Nature, 2020

The funding for the article is corrected to:

Open access funding provided by Università degli Studi di Firenze within the CRUI-CARE Agreement.

The original article has been corrected.

\footnotetext{
* Contribution to the Topical Issue "Quantum Technologies for Gravitational Physics", edited by Tanja Mehlstäubler, Yanbei Chen, Guglielmo M. Tino and Hsien-Chi Yeh.

** The online version of the article can be found at https://doi.org/10.1140/epjd/e2020-10184-6

${ }^{a}$ e-mail: francesco.marin@unifi.it
} 